\title{
Non-monotonic effects of GABAergic synaptic inputs on neuronal firing
}

\author{
Aghil Abed Zadeh ${ }^{1, *}$, Brandon D. Turner ${ }^{2}$, Nicole Calakos ${ }^{1,2,3,4}$, Nicolas Brunel ${ }^{1,4,5}$, \\ 1 Department of Neurobiology, Duke University Medical Center, Durham, NC, USA \\ 2 Department of Neurology, Duke University Medical Center,Durham, NC, USA \\ 3 Department of Cell Biology, Duke University Medical Center,Durham, NC, USA \\ 4 Duke Institute for Brain Sciences, Duke University, Durham, NC, USA \\ 5 Department of Physics, Duke University, Durham, NC, USA \\ * aghil.abed.zadeh@duke.edu
}

\begin{abstract}
GABA is canonically known as the principal inhibitory neurotransmitter in the nervous system, usually acting by hyper-polarizing membrane potential. However, GABAergic currents can also exhibit non-inhibitory effects, depending on the brain region, developmental stage or pathological condition. Here, we investigate the diverse effects of GABA on the firing rate of several single neuron models, using both analytical calculations and numerical simulations. We find that the relationship between GABAergic synaptic conductance and output firing rate exhibits three qualitatively different regimes as a function of GABA reversal potential, $v_{\mathrm{GABA}}$ : monotonically decreasing for sufficiently low $v_{\mathrm{GABA}}$ (inhibitory), monotonically increasing for $v_{\mathrm{GABA}}$ above firing threshold (excitatory); and a non-monotonic region for intermediate values of $v_{\mathrm{GABA}}$. In the non-monotonic regime, small GABA conductances have an excitatory effect while large GABA conductances show an inhibitory effect. We provide a phase diagram of different GABAergic effects as a function of GABA reversal potential and glutamate conductance. We find that noisy inputs increase the range of $v_{\mathrm{GABA}}$ for which the non-monotonic effect can be observed. We also construct a micro-circuit model of striatum to explain observed effects of GABAergic fast spiking interneurons on spiny projection neurons, including non-monotonicity, as well as the heterogeneity of the effects. Our work provides a mechanistic explanation of paradoxical effects of GABAergic synaptic inputs, with implications for understanding the effects of GABA in neural computation and development.
\end{abstract}

\section{Author summary}

Neurons in nervous systems mainly communicate at chemical synapses by releasing neurotransmitters from the presynaptic side that bind to receptors on the postsynaptic side, triggering ion flow through cell membrane and changes in the membrane potential of the postsynaptic neuron. Gamma-aminobutyric acid (GABA) is the principal neurotransmitter expressed by inhibitory neurons. Its binding to GABAergic receptors mainly causes a flow of chloride ions through the membrane, and typically hyperpolarizes the postsynaptic neuron, resulting in firing suppression. While GABA is canonically viewed as an inhibitory neurotransmitter, non-inhibitory effects have been observed in early stages of development, in stress-related disorders, and in specific parts 
of brain structures 1 14. Here, we employ analytical and computational approaches on spiking neuronal models to investigate the mechanisms of diverse effects of GABAergic synaptic inputs. We find that in addition to monotonically excitatory or monotonically inhibitory effects, GABAergic inputs show non-monotonic effects, for which the effect depends on the strength of the input. This effect is stronger in the presence of noise, and is observed in different models both at the single cell, and at the network level. Our findings provide a mechanistic explanation of several paradoxical experimental observations, with potential implications in neural network dynamics and computation.

\section{Introduction}

GABA is the principal inhibitory neurotransmitter in the nervous system. In adult animals, GABA usually suppresses action potentials in target neurons by hyperpolarizing the membrane potential. This hyperpolarization is mediated by GABA receptor channels, that are permeable to $\mathrm{Cl}^{-}$and $\mathrm{HCO}_{3}^{-}$ions. The flux of these ions usually causes a decrease in membrane potential upon GABA channels opening [5, 6].

GABAergic synapses are canonically viewed as inhibitory. However, multiple studies have found that GABA can have non-inhibitory effects. For instance, excitation mediated by GABA plays an important role in early phases of development and neural integration during neurogenesis. It has been shown that this excitatory effect is caused by depolarizing effect of GABA on neurons $[1,7,10]$. Other studies suggest that pathological conditions of stress or trauma can lead to excitatory effects of GABA [2,11. Moreover, even in healthy adult animals, GABA mediates excitatory effects in certain brain regions. This excitatory effect of GABA has been observed in several brain regions including cortex, basal ganglia, thalamus and cerebellum [4 12 16]. In addition to excitatory effects of GABA, other studies have shown non-monotonic effects of GABA, suggesting that GABAergic synaptic currents produce excitation or inhibition based on their strength. In hippocampal interneurons, it has been shown that tonic GABA conductance affects neuronal firing in a non-monotonic way [17]. In the striatum, bidirectional optogenetic manipulations (inhibition and excitation) of fast spiking interneurons (FSIs) cause spiny projection neurons (SPNs) population activity inhibition [18, Fig 1], suggesting that SPNs activity may depend on FSI GABAergic inputs in a non-monotonic way. In the non-monotonic cases, small GABAergic inputs promote neural firing while large currents have an inhibitory effect. The non-inhibitory effects of GABA significantly influence network dynamics and have been shown to affect neural synchrony and rhythmic activity 19,20 . Understanding the mechanisms of such effects in neural dynamics is central to unravel their role in neural computation and plasticity and combat related diseases.

In this paper, we investigate potential mechanisms of different GABA effects in neuronal and circuit models. We show how changing GABA reversal potential affects the firing rate of neurons. In particular, we show that GABA can be inhibitory, non-monotonic or excitatory depending on its reversal potential. The non-monotonic regime is observed when GABA reversal potential is below, but close to the firing threshold. Using analytical calculations in a leaky integrate and fire (LIF) conductance-based model, we provide a phase diagram of the dynamics and analyze it in the presence of input noise, showing that noisy input expands the non-monotonic regime. We also investigate a more realistic model that describes more accurately the electrophysiological properties of SPNs in the striatum, to check the robustness of our model. Finally, we study a network model of striatum to explain several observed paradoxical effects of GABAergic currents of striatal FSIs. The motivation to focus on striatum is the observation of multiple effects of FSIs on their SPN targets that cannot be explained by pure excitation nor inhibition [14,18,21]. Furthermore, it is known that 
striatal FSIs influence behavioral outputs, and a deficit in these cells has been observed in dystonia and Tourette syndrome [22]. We use network simulations and computational analysis to provide possible insights into non-monotonic and heterogeneous effects of GABAergic inputs on their targets. These analyses can be used to address several experimental findings and to provide a framework for understanding the role of GABA in network dynamics and plasticity in different brain regions or developmental stages.

\section{Materials and methods}

We use three different models to study the effects of GABAergic synaptic inputs on neuronal firing rate. We start by analyzing a single-cell conductance-based LIF model which is analytically tractable. We then expand our computational analysis on a more realistic neuronal model. Finally, we implement and analyze a micro-circuit model of striatum.

\section{Leaky integrate-and-fire model}

This model describes the dynamics of the membrane potential which is given by a current balance equation, with capacitive and leak currents, together with conductance-based GABAergic and glutamatergic synaptic currents. Additionally a stochastic Gaussian noise is used to study the effect of noisy inputs. The model can be described by a small number of parameters, and at the same time captures different effects of GABAergic conductance on firing activity. The evolution of the membrane potential follows

$$
\tau \frac{d v}{d t}=-\left(v-v_{\mathrm{L}}\right)-g_{\mathrm{GABA}}\left(v-v_{\mathrm{GABA}}\right)-g_{\mathrm{Glu}}\left(v-v_{\mathrm{Glu}}\right)+I_{\text {noise }}(t)
$$

when the membrane potential is smaller than a threshold, $v<v_{\text {thr }}$. Spikes are emitted whenever $v=v_{\text {thr }}$, after which the voltage is reset instantaneously to $v_{\text {reset }}$. In Eq (1), $\tau$ is the membrane time constant (ratio of capacitance and leak conductance); $v_{\mathrm{L}}, v_{\mathrm{GABA}}$ and $v_{\mathrm{Glu}}$ are the leak, GABA and glutamate reversal potentials respectively; $g_{\mathrm{GABA}}$ and $g_{\text {Glu }}$ are the ratio of GABAergic and glutamatergic conductances to the leak conductance. Throughout the paper, we use $\tau=20 \mathrm{~ms}, v_{\mathrm{L}}=-80 \mathrm{mV}, v_{\mathrm{Glu}}=0 \mathrm{mV}$, $v_{\mathrm{thr}}=-60 \mathrm{mV}$ and $v_{\text {reset }}=-70 \mathrm{mV}$. These parameters are set to be in the range of experimentally observed values, and the reported results are qualitatively robust to changes of these parameters in such a range. The last term in the R.H.S. of Eq (1) is a noise term, $I_{\text {noise }}=\sigma \sqrt{\tau} \zeta(t)$ in which $\zeta$ is a Gaussian white noise with unit variance. We first analyze the dynamics in the absence of noise $(\sigma=0)$ and then investigate the effects of noise on the dynamics. We study two different classes of noise. First, we consider additive noise in which $\sigma$ is a constant, independent of membrane potential and conductances. Second, we study noise that represents fluctuations caused by Poisson firing of presynaptic neurons, in which case, $\sigma$ depends on several model parameters and membrane potential.

\section{EIF-KIR model}

While most of our analysis is based on the model of Eq (1), we also analyze a more realistic neuronal model to show the qualitative robustness of our results. In this model, as represented in Eq (2), two more currents are added: an exponential current $\left(I_{\mathrm{EXP}}\right) 23$ that represents in a simplified fashion the fast sodium currents near the spiking threshold, and an Inward-Rectifier potassium current $\left(I_{\mathrm{KIR}}\right)$ that captures the 
nonlinear dependence of effective conductance of membrane potential. The dynamics of this EIF-KIR model is given by

$$
\begin{aligned}
& \tau \frac{d v}{d t}=-\left(v-v_{\mathrm{L}}\right)-g_{\mathrm{GABA}}\left(v-v_{\mathrm{GABA}}\right)-g_{\mathrm{Glu}}\left(v-v_{\mathrm{Glu}}\right)+I_{\mathrm{EXP}}(v)+I_{\mathrm{KIR}}(v) \\
& I_{\mathrm{EXP}}(v)=\Delta_{\mathrm{T}} \exp \left(\frac{v-v_{\mathrm{T}}}{\Delta_{\mathrm{T}}}\right) \\
& I_{\mathrm{KIR}}(v)=g_{\mathrm{K}} /\left(1+\exp \left(\frac{v-v_{\mathrm{K}}}{k}\right)\right)\left(v-v_{\mathrm{K}}\right)
\end{aligned}
$$

The parameters of the model are chosen such that it reproduces sub-threshold (current-voltage relation) and supra-threshold (current-firing rate relation) properties of a typical spiny projection neuron in striatum (see Fig 5 -d and refs 24 ). Here, we use $\Delta_{\mathrm{T}}=2 \mathrm{mV}, v_{\mathrm{T}}=-60 \mathrm{mV}, g_{\mathrm{K}}=5, k=16 \mathrm{mV}$ and $v_{\mathrm{K}}=v_{\mathrm{L}}=-80 \mathrm{mV}$, if not specified. In this model, there is no hard spike threshold, rather the membrane potential resets when the voltage diverges to infinity due to the exponential spike-generating current. To calculate V-I and f-I curves, we add a constant current of form $I_{\text {const }}=I / g_{L}$ to Eq $(1 \mathrm{p})$ in which $I$ is in the units of $\mathrm{pA}$ and we set $g_{L}=5 \mathrm{nS}$.

\section{Circuit model}

We used a micro-circuit model of a striatal network to demonstrate paradoxical effects of GABA at the network level. Single neurons were described by Eq (1) with only leak, GABAergic and glutamatergic currents. There are three types of neurons in the model: direct and indirect spiny projection neurons (dSPN \& iSPN) and fast spiking interneurons (FSI). The network is composed of $N=1000$ neurons with $2 \%$ FSI, $49 \%$ $\mathrm{dSPN}$ and $49 \%$ iSPN, approximating a simplified micro-circuit of $\sim 0.01 \mathrm{~mm}^{3}$ of mouse striatum 24, 25. FSIs have a GABA reversal potential of $-80 \mathrm{mV}$ and provide feedforward GABAergic currents to SPNs. The glutamatergic conductances are taken for simplicity to be driven by independent Poisson inputs convoluted with an exponential kernel and a frequency of $1000 \mathrm{~Hz}$, approximating the total cortical input to a striatal neuron. The exponential kernel has a decay time constant of $5.6 \mathrm{~ms}$ (similar to [26]). The mean value of this Poisson excitatory input is chosen to reproduce the desired firing rates in the model. Neurons are connected by GABAergic synapses in which a spike in presynaptic neuron produces a postsynaptic conductance of form $g_{\mathrm{GABA}}(t)=g_{0}\left(\exp \left(t / \tau_{1}\right)-\exp \left(t / \tau_{2}\right)\right)$ with rise time of $\tau_{1}=1.5 \mathrm{~ms}$ and decay time of $\tau_{2}=20 \mathrm{~ms}$ [27]. The connectivity probabilities and strengths are inferred from several experiments [27,28] and are represented in Eq (3) in which $P_{i j}$ and $G_{i j}$ corresponds to connection probabilities and postsynaptic conductance strength of a spike $\left(g_{0}\right)$ from population $j$ to population $i$ with indices $1,2,3$ standing for FSIs, dSPNs and iSPNs respectively.

$$
P=\left[\begin{array}{ccc}
0.58 & 0 & 0 \\
0.53 & 0.26 & 0.27 \\
0.36 & 0.06 & 0.36
\end{array}\right] \quad G=\left[\begin{array}{ccc}
0.06 & 0 & 0 \\
0.5 & 0.04 & 0.13 \\
0.5 & 0.11 & 0.11
\end{array}\right]
$$

Note that $G$ entries are dimensionless, being a ratio of synaptic to leak conductances, as $g_{\mathrm{GABA}}$ in $\mathrm{Eq}(2)$. In simulations, the first $100 \mathrm{~ms}$ are removed to avoid transient effects.

\section{Results}

\section{Effects of GABA on the deterministic LIF model}

To gain insight into the mechanisms of different effects of GABA, we start analyzing Eq (1) without the noise term and with constant synaptic conductances. This case 
represents a steady state neuron receiving constant GABAergic and glutamatergic currents and facilitates analytic calculations. With these assumptions, the dynamics of the membrane potential become deterministic and can be rewritten as

$$
\tau \frac{d v}{d t}=-g_{\mathrm{eff}}\left(v-v_{\mathrm{eff}}\right)
$$

where the effective input conductance $g_{\text {eff }}$ (relative to the leak) and the effective reversal potential $v_{\text {eff }}$ are given by

$$
\begin{aligned}
& g_{\mathrm{eff}}=1+g_{\mathrm{GABA}}+g_{\mathrm{Glu}} \\
& v_{\mathrm{eff}}=\frac{v_{\mathrm{L}}+g_{\mathrm{GABA}} v_{\mathrm{GABA}}+g_{\mathrm{Glu}} v_{\mathrm{Glu}}}{1+g_{\mathrm{GABA}}+g_{\mathrm{Glu}}}
\end{aligned}
$$

According to Eq (4), the membrane potential relaxes exponentially to an effective potential, $v_{\text {eff }}$, with an effective time constant $\tau_{\text {eff }}=\tau / g_{\text {eff }}$. If the effective potential is greater than $v_{\mathrm{thr}}$, the neuron spikes with a non-zero firing rate $\nu$, given by

$$
\nu=\frac{g_{\text {eff }}}{\tau \log \left(\frac{v_{\text {eff }}-v_{\text {reset }}}{v_{\text {eff }}-v_{\text {thr }}}\right)}
$$

Fig 1 shows examples of how the firing rate depends on $g_{\mathrm{GABA}}$ for different values of $g_{\mathrm{Glu}}$ and $v_{\mathrm{GABA}}$. As shown in Fig $1 \mathrm{l}, \mathrm{b}$, the firing rate vs GABA conductance curves can be decreasing, non-monotonic or increasing, depending on $v_{\mathrm{GABA}}$ and $g_{\mathrm{Glu}}$. When $v_{\mathrm{GABA}}>v_{\mathrm{thr}}$, GABAergic current is always excitatory. When $v_{\mathrm{GABA}}<v_{\mathrm{thr}}$, large $g_{\mathrm{GABA}}$ leads to inhibition; however, it can be excitatory for smaller values of $g_{\mathrm{GABA}}$.

When $v_{\mathrm{GABA}}<v_{\mathrm{thr}}$, large enough $g_{\mathrm{GABA}}$ silences the neuron. The GABAergic conductance for which the firing stops, $g_{\mathrm{GABA}}^{s}$, can be calculated using the condition $v_{\text {eff }}=v_{\text {thr }}$. This leads to

$$
g_{\mathrm{GABA}}^{s}=\frac{\left(v_{\mathrm{thr}}-v_{\mathrm{L}}\right)+g_{\mathrm{Glu}}\left(v_{\mathrm{thr}}-v_{\mathrm{Glu}}\right)}{v_{\mathrm{GABA}}-v_{\mathrm{thr}}} .
$$

Fig 1; , d shows the collapse of zero-firing onset when $g_{\mathrm{GABA}}$ is scaled appropriately with respect to $v_{\mathrm{GABA}}$ and $g_{\mathrm{Glu}}$. This onset increases with $g_{\mathrm{Glu}}$ and $v_{\mathrm{GABA}}$ and diverges to infinity as $v_{\mathrm{GABA}} \rightarrow v_{\mathrm{thr}}$.

The occurrence of the different regimes of GABA effects on firing rate can be understood by analyzing Eq (7). In this equation, $g_{\mathrm{GABA}}$ acts on the firing rate in two ways: Through its effects on $g_{\text {eff }}$, and on $v_{\text {eff }}$. Increasing GABA conductance increases $g_{\text {eff }}$, which tends to increase the firing rate due to the decrease in effective membrane time constant of the neuron, while the effect of GABA on $v_{\text {eff }}$ depends on the GABA reversal potential. The competition between these two effects can be analyzed by computing the derivative of the firing rate with respect to the GABA conductance,

$$
\frac{d \nu}{d g_{\mathrm{GABA}}}=\frac{\nu}{g_{\mathrm{eff}}}\left(1+\left(v_{\mathrm{GABA}}-v_{\mathrm{eff}}\right) \frac{\left(v_{\mathrm{thr}}-v_{\text {reset }}\right)}{\left(v_{\mathrm{eff}}-v_{\text {reset }}\right)\left(v_{\mathrm{eff}}-v_{\mathrm{thr}}\right)} \frac{\tau \nu}{g_{\mathrm{eff}}}\right)
$$

where the first and second terms within the parenthesis represent the effects of $g_{\text {eff }}$ and $v_{\text {eff }}$ on the firing rate, respectively. The sign of this derivative determines whether GABAergic conductance is inhibitory or excitatory. The sufficient conditions for non-monotonic effect can be summarized as:

(a) $\left.\frac{d \nu}{d g_{\mathrm{GABA}}}\right|_{g_{\mathrm{GABA}}=0}>0$

(b) $\quad v_{\mathrm{GABA}}<v_{\mathrm{thr}}$ 
a

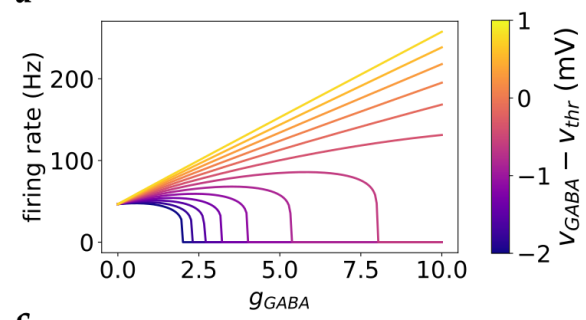

c

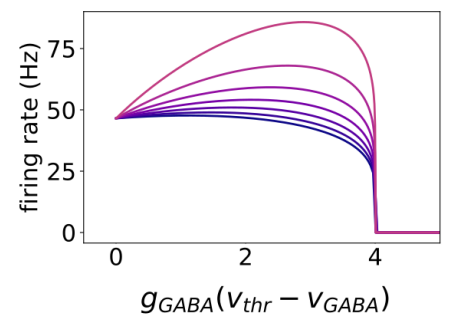

b

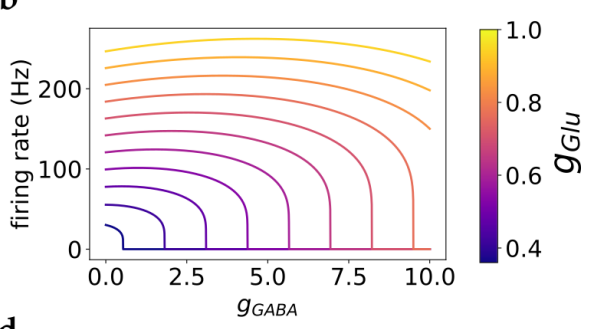

d

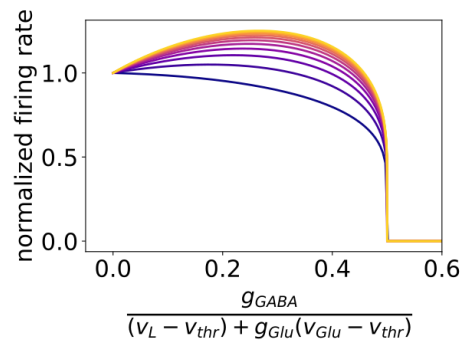

e

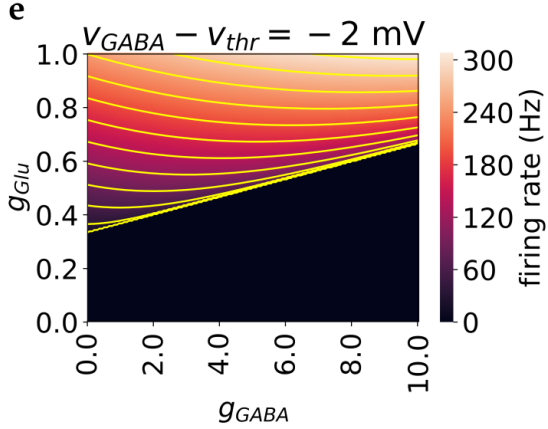

Fig 1. Effect of $g_{\text {GABA }}$ on firing rate in the deterministic LIF model. a) firing rate $\nu$, as a function of GABAergic conductance for different GABA reversal potentials $\left(g_{\mathrm{Glu}}=0.4\right)$. When GABA reversal potential is below and close to firing threshold, $v_{\mathrm{thr}}$, the firing rate has a non-monotonic dependence on $g_{\mathrm{GABA}}$. b) Firing rate vs $g_{\mathrm{GABA}}$ for different $g_{\mathrm{Glu}},\left(v_{\mathrm{GABA}}-v_{\mathrm{thr}}=-3 \mathrm{mV}\right)$. The non-monotonicity appears when the glutamatergic conductance is sufficiently large. c) Firing rate vs GABA conductance rescaled with $v_{\mathrm{thr}}-v_{\mathrm{GABA}}$, for $v_{\mathrm{thr}}>v_{\mathrm{GABA}}$. For GABA reversal potentials smaller than the threshold, complete inhibition occurs for large GABA conductances. The onset of zero-firing scales with $1 /\left(v_{\mathrm{thr}}-v_{\mathrm{GABA}}\right)$ as shown by the collapsed curves (same color code as above). d) Firing rate vs rescaled $g_{\mathrm{GABA}}$. The collapsed curves show how the zero-firing onset scales with $g_{\text {Glu }}$ (same color code as above). e) Heatmap of firing rate as a function of GABAergic and glutamatergic conductances for $v_{\mathrm{GABA}}-v_{\mathrm{thr}}=-2 m V$. The yellow curves are contours lines of constant firing rates. 
a

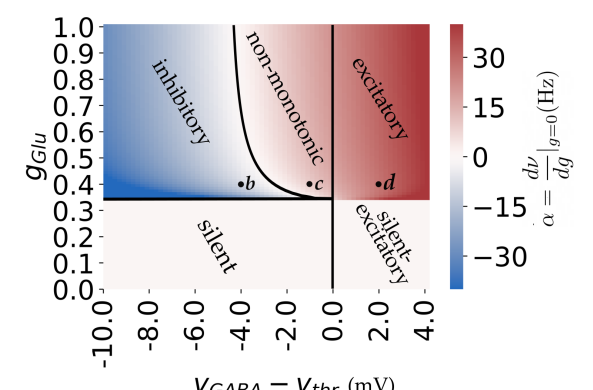

e

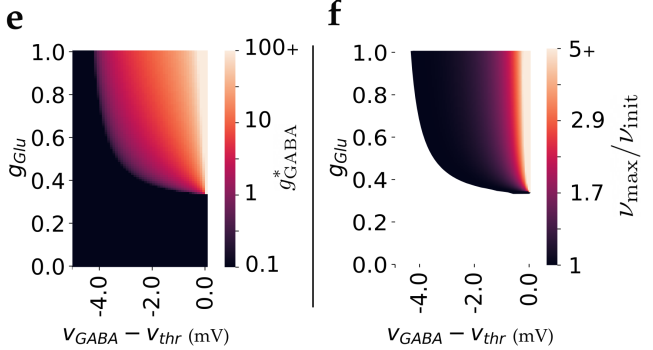

b

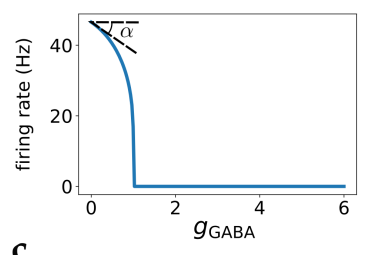

C

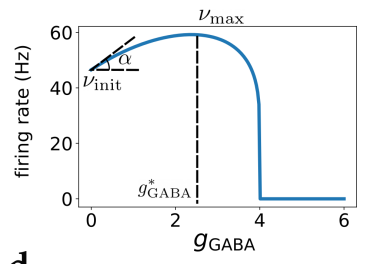

d

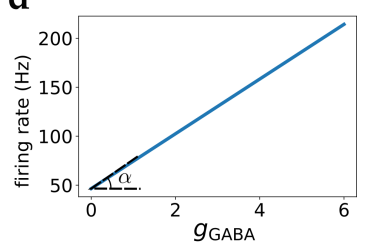

Fig 2. GABA effect phase diagram in the deterministic LIF model. a) Phase diagram of GABA effects. The colorbar indicates the initial slope of firing curves with respect to $g \equiv g_{\mathrm{GABA}}$ as shown in (b-d). b, c, d marked in the phase diagram correspond to three example firing rate curves below. b,c,d) As $v_{\mathrm{GABA}}$ increases, GABA effect changes from inhibitory to excitatory with a non-monotonic region in between. In this region, GABA conductance below a certain threshold, $g_{\mathrm{GABA}}^{*}$, has an excitatory effect while large GABA conductances have an inhibitory effect. e) $g_{\mathrm{GABA}}^{*}$ (GABA conductance that maximizes output firing rate, shown in part c) in the non-monotonic region of phase diagram. Colorbar is in logarithmic scale. f) Ratio of maximum firing rate to firing rate at $g_{\mathrm{GABA}}=0\left(\nu_{\max } / \nu_{\text {init }}\right.$ shown in part c $)$ in the non-monotonic region, as a measure of non-monotonic effect strength. Colorbar is in logarithmic scale. 
Condition (a) makes GABA excitatory for small $g_{\mathrm{GABA}}$ and the condition (b) makes GABA inhibitory for large $g_{\text {GABA }}$. These conditions can be met when the term $v_{\text {eff }}-v_{\mathrm{GABA}}$ in Eq 8 is positive but not too large, which means $v_{\text {eff }}$ should be above and close to $v_{\mathrm{GABA}}$. The critical value of $v_{\mathrm{GABA}}$ that separates the inhibitory and non-monotonic regime, $v_{\mathrm{GABA}}^{*}$, can be obtained from Eq (8):

$$
v_{\mathrm{GABA}}^{*}=v_{\text {eff }}-\frac{\left(v_{\text {eff }}-v_{\text {reset }}\right)\left(v_{\text {eff }}-v_{\text {thr }}\right)}{\left(v_{\text {thr }}-v_{\text {reset }}\right)} \ln \left(\frac{v_{\text {eff }}-v_{\text {reset }}}{v_{\text {eff }}-v_{\text {thr }}}\right)
$$

For small $g_{\mathrm{Glu}}, v_{\mathrm{eff}} \approx v_{\mathrm{thr}}$ which leads to $v_{\mathrm{GABA}}^{*}=v_{\mathrm{thr}}$. On the other hand, for large $g_{\mathrm{Glu}}, v_{\mathrm{eff}} \gg v_{\mathrm{thr}}$, which results in $v_{\mathrm{GABA}}^{*}$ converging to $\left(v_{\text {reset }}+v_{\mathrm{thr}}\right) / 2$.

These results are shown in the phase diagram of Fig $2 \mathrm{a}$. This phase diagram separates the $v_{\mathrm{GABA}}-g_{\mathrm{Glu}}$ plane into five regions: For sufficiently high $g_{\mathrm{Glu}}$ (above the horizontal line in Fig $2 \mathrm{a}$ ), GABA is always inhibitory for $v_{\mathrm{GABA}}<v_{\mathrm{GABA}}^{*}$ (Fig 2p); The GABA effect is non-monotonic for $v_{\mathrm{thr}}>v_{\mathrm{GABA}}>v_{\mathrm{GABA}}^{*}$ (Fig 20); GABA is always excitatory for $v_{\text {thr }}<v_{\text {GABA }}($ Fig $2 \mathrm{~d})$. Finally, for low $g_{\text {Glu }}$ (below the horizontal line in Fig $2 \mathrm{a}$ ), the neuron either remains silent for all GABA conductances for $v_{\mathrm{GABA}}<v_{\mathrm{thr}}$, or starts firing with an increasing rate beyond a critical value of the GABA conductance for $v_{\mathrm{GABA}}>v_{\mathrm{thr}}$. Fig $2 \mathrm{a}$ also shows how the slope of the firing rate vs $g_{\mathrm{GABA}}$ curve at zero GABA conductance, i.e. $\frac{d \nu}{d g_{\mathrm{GABA}}}$, depends on both glutamatergic conductance and GABA reversal potential.

Fig 2 , f provide additional characterizations of the non-monotonic regime. Fig 2 shows how the GABA conductance that maximizes the firing rate depends on $v_{\mathrm{GABA}}$ and $g_{\mathrm{Glu}}$. The colorbar represents $g_{\mathrm{GABA}}^{*}$ as shown in Fig 20xample. Fig 2f shows the strength of the non-monotonic effect, i.e. the ratio between maximal firing rate $\nu_{\max }$ and firing rate in the absence of GABA $\nu_{\text {init }}$ in the non-monotonic region. Here $\nu_{\text {init }}=\nu\left(g_{\mathrm{GABA}}=0\right)$ and $\nu_{\max }=\max \left(\nu\left(g_{\mathrm{GABA}}\right)\right)$. This ratio converges to 1 when $v_{\mathrm{GABA}} \rightarrow v_{\mathrm{GABA}}^{*}$, and diverges in the limit that $v_{\mathrm{GABA}} \rightarrow v_{\mathrm{thr}}$.

To conclude, in the deterministic LIF model, there is a region of parameters for which GABA has a non-monotonic effect on neuronal firing rate, when $v_{\mathrm{GABA}}$ is below but sufficiently close to $v_{\mathrm{GABA}}$. However, this effect is weak except in a narrow range of $v_{\mathrm{GABA}}$ close to threshold, as shown in Fig $2 \mathrm{f}$.

\section{Input noise expands the non-monotonic regime}

So far, we have investigated the effect of constant deterministic GABAergic synaptic inputs. In real neurons, the synaptic currents are noisy for multiple reasons, including stochastic vesicle release and channel opening. In addition, presynaptic neuronal firing can often exhibit a large degree of irregularity, which is typically approximated by a Poisson process. The total synaptic currents to a neuron can then be described by the sum of their temporal mean, and stochastic fluctuations around the mean. When synaptic inputs are independent, Poissonian and with a time constant that are much smaller than the membrane time constant, these fluctuations can be approximated by white noise. In this section, we turn to an investigation of the effects of white noise on how GABA affects neuronal firing rate.

The firing rate of the neuronal model of Eq (1) in the presence of noise is given by 29,30 :

$$
\begin{aligned}
& \nu=\left[\tau_{\text {eff }} \sqrt{\pi} \int_{x_{\text {min }}}^{x_{\text {max }}} \exp \left(x^{2}\right)(1+\operatorname{erf}(x)) d x\right]^{-1} \\
& x_{\text {min }}=\frac{v_{\text {reset }}-v_{\text {eff }}}{\sigma_{\text {eff }}}, x_{\text {max }}=\frac{v_{\text {thr }}-v_{\text {eff }}}{\sigma_{\text {eff }}} \\
& \tau_{\text {eff }}=\frac{\tau}{g_{\text {eff }}}, \sigma_{\text {eff }}=\frac{\sigma}{\sqrt{g_{\text {eff }}}}
\end{aligned}
$$


a

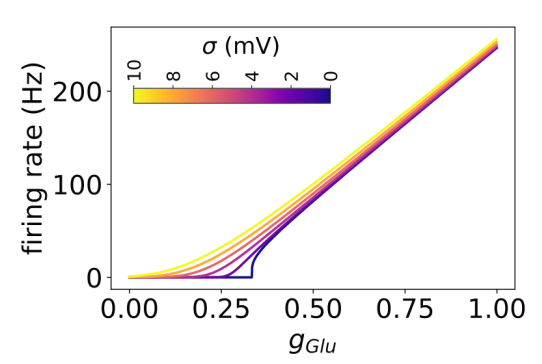

e
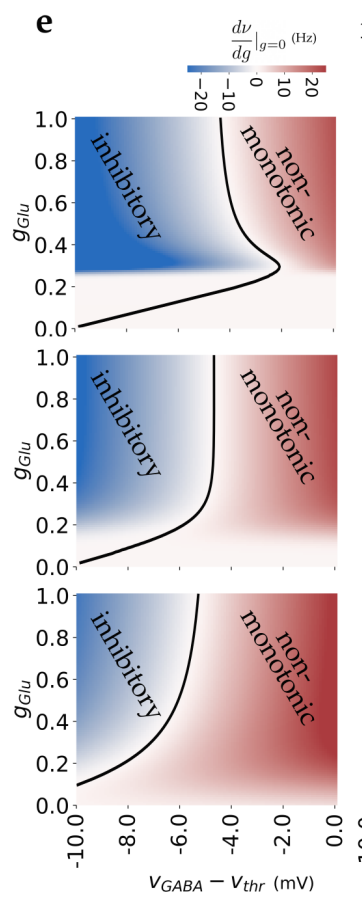

b

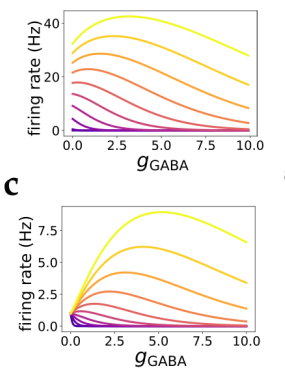

f
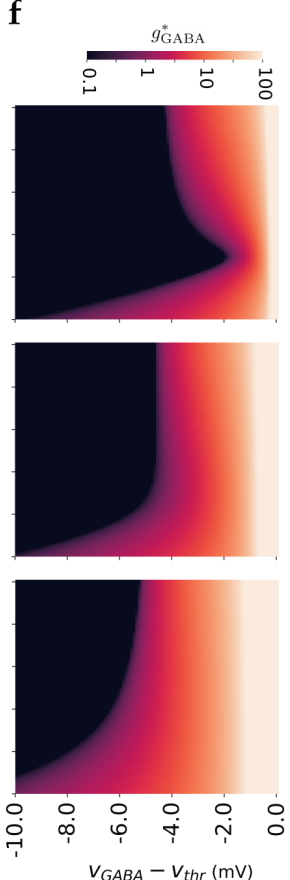

d

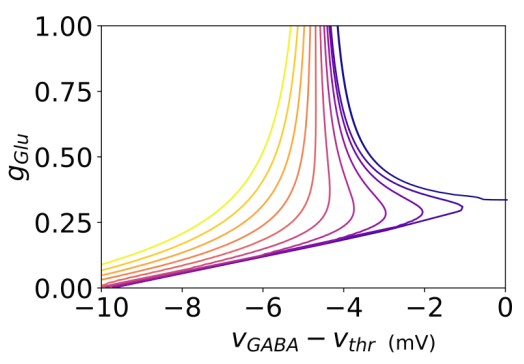

g
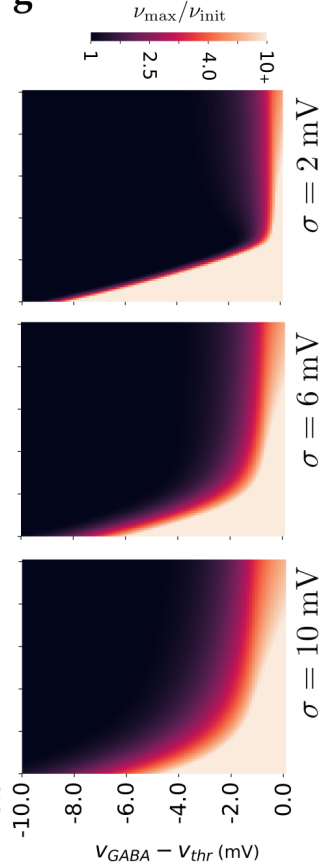

Fig 3. Effect of current-based input noise. a) Transfer functions for different noise levels. Increasing noise smooths the transfer function for low firing rates and results in firing for subthreshold values of $g_{\mathrm{Glu}}$. b) Effect of $g_{\mathrm{GABA}}$ on firing rate for different noise levels $\left(g_{\text {Glu }}=0.25\right)$. Increasing noise level, while keeping $g_{\text {Glu }}$ constant, increases both the firing rate and the non-monotonic effect. c) Effect of $g_{\text {GABA }}$ on firing rate for different noise levels $\left(\nu_{\text {init }}=1 \mathrm{~Hz}\right)$. Here $g_{\text {Glu }}$ is modified for different values of $\sigma$ to get same initial firing rate. Increasing noise in this case also strengthens the non-monotonic effect. d) Boundary between the non-monotonic and inhibitory regions of phase diagrams for different noise levels. Increasing noise extends the non-monotonic region for all values of $g_{\text {Glu }}$, with a more drastic change for smaller $g_{\text {Glu }}$. e) Examples of phase diagrams similar to that of Fig 2 for different noise levels. Introducing noise removes the silent region and enlarges the non-monotonic region. f) Similar phase diagrams as in part e, showing $g_{\mathrm{GABA}}^{*} \cdot \mathrm{g}$ ) Similar phase diagrams as in part e, showing the ratio of max firing rate to initial firing rate. 
We start by considering the case when $\sigma$ is independent of other parameters. Fig 3 a shows the firing rate as a function of $g_{\text {Glu }}$ for different values of $\sigma$ (color-coded) and $g_{\mathrm{GABA}}=0$. When there is no noise, $\sigma=0$, the firing rate is zero below a certain threshold of $g_{\mathrm{Glu}}$, increases sharply near this threshold and finally converges to a linear relation (note that the lack of saturation is due to our choice of setting the refractory period to zero). As $\sigma$ increases, the curves become smoother with the neuron firing for sub-threshold values of $g_{\text {Glu }}$, due to fluctuations of membrane potential. This effect allows the neuron to have low firing rates for a wider range of $g_{\mathrm{Glu}}$, compatible with the observed low firing rate in vivo in many brain regions $(\sim 1 \mathrm{~Hz})$. The effect of noise on the different regimes of how GABA affects neuronal firing rate are illustrated in Fig $3 \mathrm{~b}$,c. Both of these figures show firing rate as a function of GABAergic conductance for different values of noise. In Fig $3 \mathrm{~b}$ all curves receive the same $g_{\mathrm{Glu}}$, so the noise increases the firing rate and non-monotonic effect simultaneously. In Fig $3 k, g_{\text {Glu }}$ is chosen such that initial firing rate is the same $\left(\nu_{\text {init }}=1 \mathrm{~Hz}\right)$ for different noise levels. This figure also shows that increasing $\sigma$ makes the curves peak value larger, thus the non-monotonic effect becomes stronger with increasing noise level.

For non-zero noise, the neuron exhibits a non-zero firing rate for any value of $g_{\mathrm{Glu}}$. Thus, the silent region of the phase diagram in Fig 2 disappears. The boundary between non-monotonic and excitatory regions remains unchanged at $v_{\mathrm{GABA}}=v_{\mathrm{thr}}$. In the case that $v_{\mathrm{GABA}}>v_{\mathrm{thr}}$, for large $g_{\mathrm{GABA}}, v_{\text {eff }} \rightarrow v_{\mathrm{GABA}}>v_{\text {thr }}$ and $g_{\mathrm{eff}} \rightarrow$ inf; using Eq (7), the firing rate diverges and GABAergic currents are always excitatory. However, noise changes the boundary that separates the inhibitory and non-monotonic regions. Fig $3 \mathrm{~d}$ shows how the boundary between inhibitory and non-monotonic regions of the phase diagram changes as $\sigma$ increases, while Fig 3 e shows examples of the phase diagram for several values of $\sigma$. Increasing $\sigma$ expands the non-monotonic region, with a stronger effect for smaller values of $g_{\text {Glu }}$. This effect can be shown mathematically using Eq 10 in the limit of large $\sigma$ and small $g_{\mathrm{Glu}}$ and $g_{\mathrm{GABA}}$. In this case, as the limits of integral tend to zero, the integrand can be approximated by 1 , resulting in:

$$
\nu \approx\left[\tau_{\text {eff }} \sqrt{\pi}\left(x_{\text {max }}-x_{\text {min }}\right)\right]^{-1}=\frac{\sigma}{\tau \sqrt{\pi}\left(v_{\text {thr }}-v_{\text {reset }}\right)} \sqrt{1+g_{\text {GABA }}}
$$

In this limit, the firing rate is an increasing function of $g_{\mathrm{GABA}}$ which shows an excitatory effect for small GABAergic conductances, as expected in the non-monotonic regime. As a result, in the presence of strong fluctuating noise, non-monotonic effect of GABA input is present for a wide range of $v_{\mathrm{GABA}}$. Fig 3 ; shows how the GABA conductance $g_{\mathrm{GABA}}^{*}$ that maximizes firing rate depends on parameters, while Fig $3 \mathrm{~s}$ shows how the strength of the non-monotonic effect depends on $v_{\mathrm{GABA}}$ and $g_{\mathrm{Glu}}$. In particular, this figure shows that there is a much larger range of these parameters for which the non-monotonic effect is strong (i.e. ratio of maximal to initial firing rate significantly higher than 1 ).

So far, we have investigated the effect of noise whose amplitude $\sigma$ is a constant and is independent of conductances. This approach reveals the qualitative effect of noise; however, here we extend our analysis by considering a more realistic representation of noise. The main source of noise in vivo is thought to have a synaptic origin, and be due to both irregular firing of presynaptic neurons and come from stochastic release of synaptic vesicles. This stochasticity causes fluctuations that depend on the strengths of inputs, i.e. stronger inputs cause higher fluctuations. Here, we assume instantaneous synaptic conductances that are a sum of delta functions:

$$
g_{s}=\tau a_{s} \sum_{j} \delta\left(t-t_{j}\right)
$$

in which $s \in\{\mathrm{Glu}, \mathrm{GABA}\}$, presynaptic spikes are generated at times $t_{j}$ leading to an instantaneous change of magnitude $a_{s}\left(v_{s}-v\right)$ in neuron's membrane potential. A 


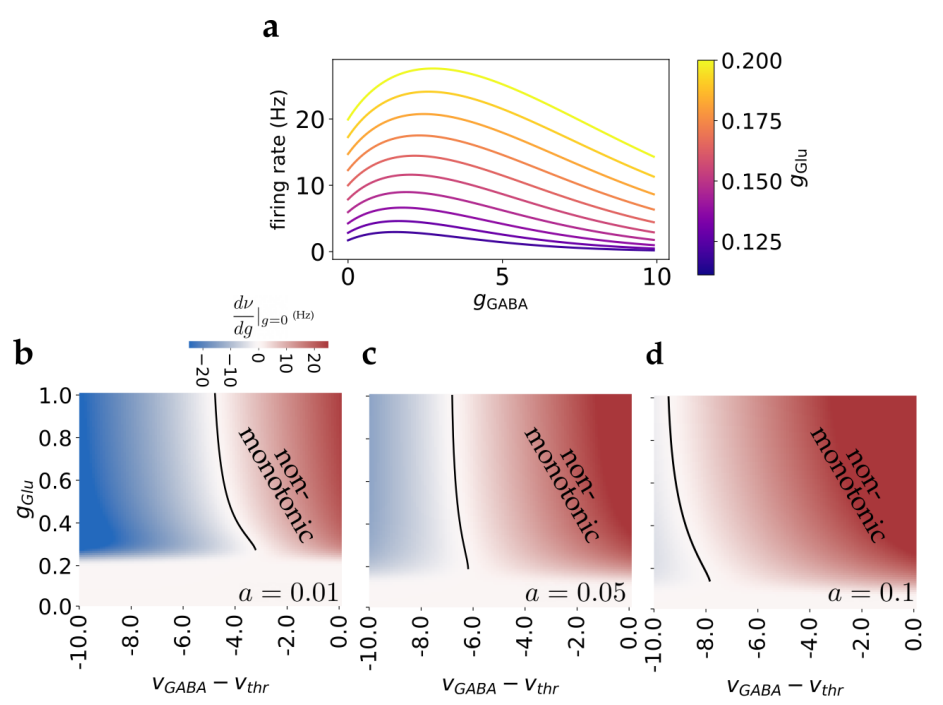

Fig 4. Effect of conductance-dependent input noise. a) Examples of firing rate vs GABAergic conductance curves, showing non-monotonic effect. Different colors represent different glutamatergic inputs. Here, $a=0.1$ and $v_{\mathrm{GABA}}-v_{\mathrm{thr}}=-5 \mathrm{mV}$. $\mathbf{b}, \mathbf{c}, \mathbf{d})$ Phase diagrams of GABA effect, similar to Fig 3 , for noise parameters $a=0.01,0.05,0.1$ respectively. Non-monotonic regime extends with increasing noise.

neuron receives synaptic inputs of type $s \in\{\mathrm{Glu}, \mathrm{GABA}\}$ from $K_{s}$ other neurons. In the limit of $K_{s} \gg 1, a_{s} \ll 1$, assuming uncorrelated Poisson firing of presynaptic neurons, synaptic inputs can be approximated by (e.g. 3032 ):

$$
\tilde{g}_{s}(t)=a_{s} \tau\left[K_{s} r_{s}+\sqrt{K_{s} r_{s}} \zeta_{s}(t)\right]
$$

in which $s$ can be Glu or GABA, $g_{s}=a_{s} \tau K_{s} r_{s}$ is the average synaptic input, $r_{s}$ is the average firing rate of connected neurons and $\zeta_{s}$ is a white Gaussian noise with zero mean and unit variance. Assuming independent glutamatergic and GABAergic synaptic spikes, the equation for the membrane voltage reduces to Eq (1) with noise term parameter:

$$
\sigma^{2}(v)=\sum_{s \in\{\mathrm{Glu}, \mathrm{GABA}\}} a_{s} g_{s}\left(v-v_{s}\right)^{2} .
$$

Here, as the noise term depends on the membrane potential $v$, it is a multiplicative noise. It can be shown however 31 that this term can be approximated by substituting $v$ by $v_{\text {eff }}, \sigma(v) \approx \sigma\left(v_{\text {eff }}\right)$, which makes the noise term independent of $v$ and easier to analyze. Using this approximation, we can obtain firing curves by applying Eq 10. Fig 4 a shows examples of firing rate vs GABA conductance curves obtained using this approximation. In this case, we set the individual synaptic strengths $a_{\mathrm{Glu}}=a_{\mathrm{GABA}}=a$. A non-monotonic effect of GABA can be observed with this model, similar to the constant noise case. The strength of noise in this model can be modulated by the value of $a$, as can be seen in Eq (12). Fig 4 4 -d provide phase diagrams of GABA effects for three different values of $a$. As can be seen in Fig 4 increasing noise expands the non-monotonic regime. This expansion is more significant for higher values of $g_{\mathrm{Glu}}$. This is in contrast to the effect of constant noise, shown in Fig 33, in which noise predominantly expands this regime for lower values of $g_{\mathrm{Glu}}$. This effect is due to the fact that in the conductance-dependent noise case, the noise term depends on the value of $g_{\mathrm{Glu}}$ and $\sigma$ vanishes as conductances go to zero, such that the noise has little effect for low values of $g_{\mathrm{Glu}}$. Overall, the results of both constant and conductance-dependent 
a
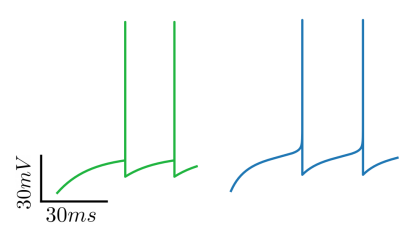

c

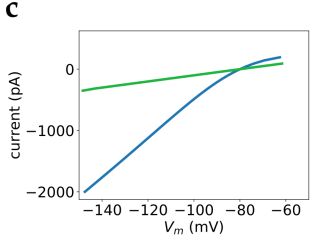

d

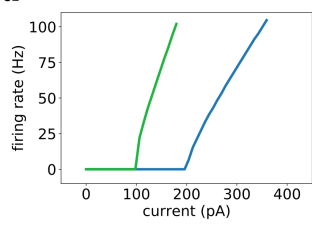

e

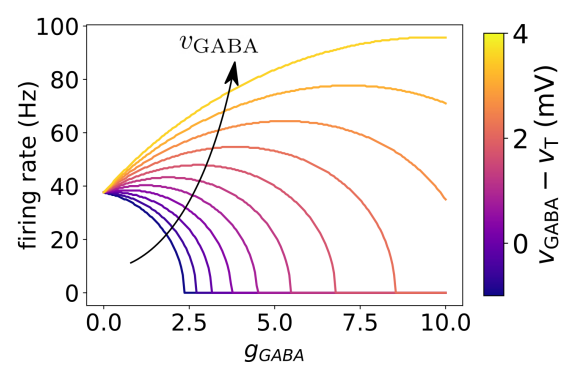

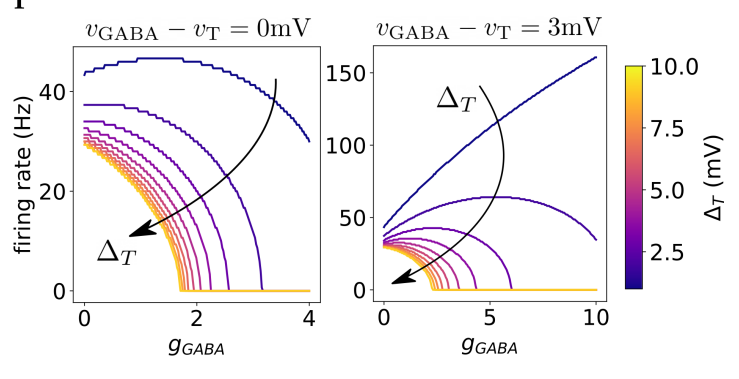

Fig 5. Dynamics of EIF-KIR neuronal model. a) Example of membrane potential dynamics of the LIF model of Eq (1) in response to a constant conductance with $g_{\text {Glu }}=0.4$. b) Example of membrane potential dynamics of EIF-KIR model of Eq (2) in response to $g_{\text {Glu }}=0.8$. This is similar to LIF model with the addition of a KIR current and a spike generating exponential current. Note the smooth dynamics leading to spike generation in the EIF-KIR model. c) V-I curve of the LIF (green) and EIF-KIR (blue) models. Note the non-linearity of the blue curve due to KIR current. d) f-I relationship of the LIF (green) and EIF-KIR (blue) models. e) Firing rate vs GABA conductance in the EIF-KIR model, for different values of GABA reversal potentials and $g_{\mathrm{Glu}}=0.8$. The non-monotonic effect is clearly observed in this model for $v_{\mathrm{GABA}}$ near $v_{\mathrm{T}}$, similar to the LIF model. f) Effect of the exponential spike generating current on non-monotonicity: Increasing $\Delta_{T}$ (Eq. (2), diminishes non-monotocity for low values of $v_{\mathrm{GABA}}$ (left) and enhances non-monotonicity for high values of $v_{\mathrm{GABA}}$ (right), effectively moving non-monotonic regime to higher values of $v_{\mathrm{GABA}}$.

noise suggest the strengthening and expansion of non-monotonic effects as input noise increases.

\section{Non-monotonic effects in a more realistic neuronal model}

To check that GABA effects are not specific to the LIF model, we analyze the dynamics of a neuronal model described by Eq (2), in which two more currents are added: An exponential current (EXP) that influences the near threshold dynamics and spike generation [23, and an inward-rectifier potassium current (KIR) that accounts for non-linear dependence of membrane conductivity as a function of membrane potential (see e.g. [33] chapter 4.4.3). Fig 5 b shows an example simulation of this model in response to a constant input conductance. The EXP term changes the near threshold dynamics and the KIR current reproduces the non-linear dependence of voltage on current, as observed in several neuronal types, including striatal neurons [34] (see Fig 5F). In this model, there is no hard spiking threshold as in Eq (1), rather the EXP term generates spikes whenever the voltage gets sufficiently close to $v_{\mathrm{T}}$, so that the EXP term leads to divergence of the voltage. Fig 5 shows the effect of GABAergic currents for different GABA reversal potentials. As shown in the figure, the non-monotonic effect is observed when the GABA reversal potential is close to $v_{\mathrm{T}}$. For $v_{\mathrm{GABA}}$ far from $v_{\mathrm{T}}$, the GABA is effectively inhibitory or excitatory, qualitatively 
similar to that of Eq (1). The non-monotonic curves of Fig 5 are slightly different compared to those of Fig 1 1 mainly because of the EXP term. The effect of the exponential spike-generating current on the non-monotonic regime can be seen in Fig 5 : As $\Delta_{T}$ in Eq (2) increases (and therefore spike generation becomes less sharp), the influence of the exponential term grows. For low values of $v_{\mathrm{GABA}}$, the non-monotonic regime becomes inhibitory, while for higher values of $v_{\mathrm{GABA}}$, the excitatory regime becomes non-monotonic. Thus, increasing $\Delta_{T}$ effectively shifts the non-monotonic regime to higher values of GABA reversal potential. Analysis of the model with the EXP term but no KIR current produces very similar curves as in Fig 5 e (not shown).

\section{Population-level effects of GABA}

In the previous sections, we investigated the effect of GABAergic currents on single neuron models. The single neuron results can be used as an approximation for steady state population dynamics in which a neuron effectively receives an input which can be expressed as sum of a constant and a white noise. In this section, we use computational modeling to analyze the effects of GABA at the population level. The model represents a striatal micro-circuit and is aimed at understanding paradoxical effects of GABAergic fast spiking interneurons (FSIs) onto spiny projection neurons (SPNs). In-vivo electrophysiological recordings in combination with optogenetic stimulation of FSIs have suggested a non-monotonic effect on SPN firing rates, in which increasing or reducing FSI activity results in SPNs inhibition [18]. The model consists of three different populations, fast spiking interneurons (FSIs), and direct and indirect spiny projection neurons (dSPNs, iSPNs). There are several other interneuron types in striatum; however, here we only include FSIs as they provide the major GABAergic inputs onto SPNs 24 .

Fig 6a presents a schematic of the model in which neurons receive uncorrelated glutamatergic Poisson inputs, representing cortical synaptic currents, and are connected by GABAergic synapses. The excitatory inputs are tuned such that the average firing rate of SPNs and FSIs are close to those recorded in awake rodents, which are about $1 \mathrm{~Hz}$ and $10 \mathrm{~Hz}$ respectively $[18$. Fig $6 \mathrm{~b}-\mathrm{c}$ show raster plots and membrane potentials of randomly selected neurons. Membrane potentials fluctuate in the sub-threshold range due to the random arrival of synaptic inputs, leading to irregular firing at low rates. The distribution of membrane potential of SPNs are presented in Fig 6 $\mathrm{d}$. It is close to a Gaussian, with a width of $1.1 \mathrm{mV}$. Fig 6 shows how SPN population mean firing rate depends on FSI population mean rate. When $v_{\mathrm{GABA}}$ is within a few $\mathrm{mV}$ of $v_{\mathrm{thr}}$, (here $1 \mathrm{mV}$ ), a non-monotonic dependence can be observed, while lower $v_{\mathrm{GABA}}$ results in an average inhibitory effect of FSIs on SPNs. Thus, in these simulations, both increasing or decreasing FSI activity from its normal firing rate $(10 \mathrm{~Hz})$ results in inhibition of SPN population, similar to in-vivo observations [18. The difference between dSPN and iSPN curves are due to asymmetric connectivity of FSIs to these two populations. As FSIs are connected to dSPNs with higher probability, the direct pathway is modulated more strongly by FSI feedforward currents. Thus, FSIs can modulate the direct-indirect pathway balance. In addition to a change in SPN mean firing rate, FSIs also affect the width of the distribution of SPN firing rates. Fig 6: shows the distributions of SPNs firing rates for two values of FSI activity, $0 \mathrm{~Hz}$ and $25 \mathrm{~Hz}$. While the mean rates are similar, the width of the distribution increases with increasing FSI activity. Thus, there is a strong heterogeneity in changes in individual SPNs firing rate, shown in Fig 6g. This heterogeneity can be explained by non-monotonic effects of FSI input onto SPNs firing rate. As shown in Fig $6 \mathrm{~h}$, SPNs with smaller number of FSI inputs increase their firing rate, while SPNs receiving strong FSI inputs decrease their rates. Thus, our network model can potentially explains why manipulations of FSI activity can result in excitatory or inhibitory effect in different subsets of SPNs 14,21$]$. 

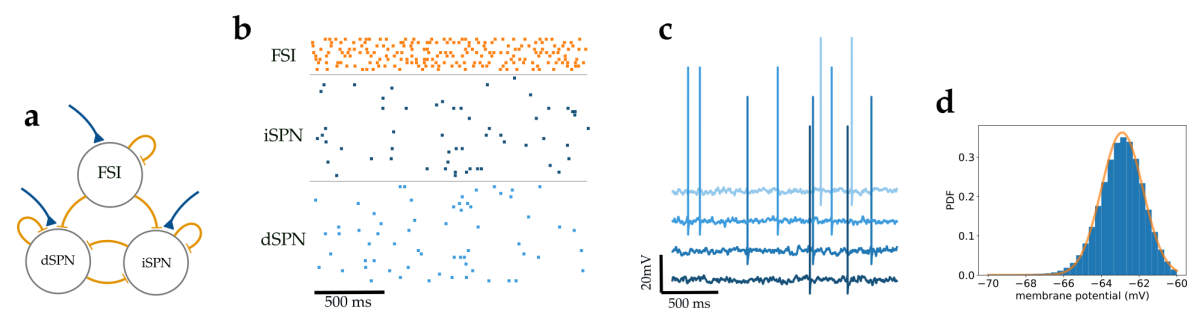

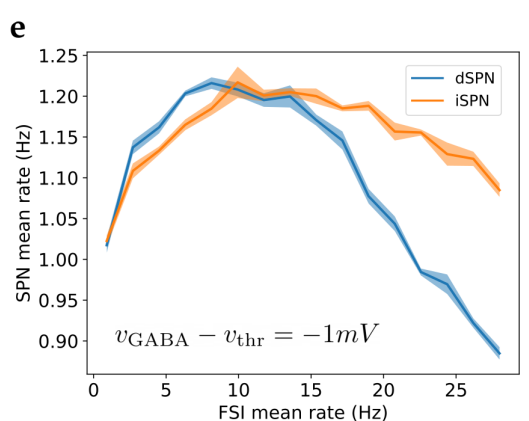

f

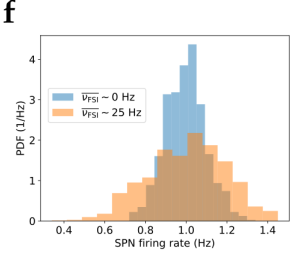

g
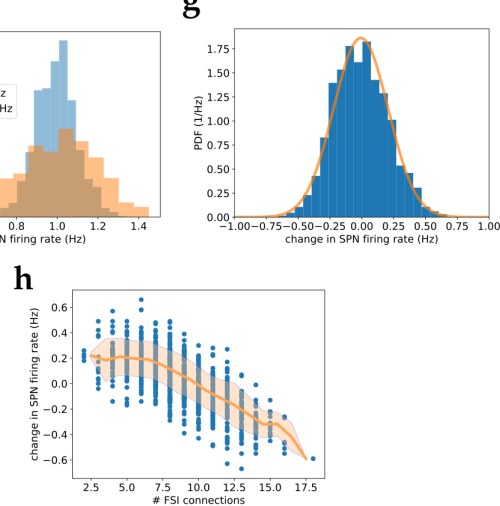

h

Fig 6. Non-monotonic effects of GABA in a striatal microcircuit model. a) Schematic of striatal micro-circuit, composed of three types of neurons, fast spiking interneurons (FSIs) and D1 and D2 spiny projection neurons (dSPNs and iSPNs representing direct and indirect pathways). All types receive glutamatergic cortical inputs (blue), and interact through GABAergic synapses (orange). b) Raster plot of randomly selected neurons from different populations. External inputs are chosen so that FSIs and SPNs fire at experimentally recorded rates in vivo $(10 \mathrm{~Hz}$ and $1 \mathrm{~Hz}$ respectively) c) Examples of membrane potentials of randomly selected SPNs. d) Probability distribution function of SPNs membrane potential. The orange line is a fitted Gaussian with a std of $1.1 \mathrm{mV}$. e) Non-monotonic response of SPN population mean firing rate as a function of FSI population mean firing rate, when $v_{\mathrm{GABA}}$ is $1 \mathrm{mV}$ below $v_{\text {thr }}$ in SPNs. The shaded area represents std around the mean, computed from a total of 100 independent network realizations. f) Distribution of SPN firing rates when FSI mean firing rate is $0 \mathrm{~Hz}$ (blue) and $25 \mathrm{~Hz}$ (orange) computed from 100s of network simulations. The mean rates are similar, while the the std of distributions are 1.1 and $1.9 \mathrm{~Hz}$ respectively. g) Distribution of the change in firing rate of individual SPNs when FSI rate changes from $0 \mathrm{~Hz}$ to $25 \mathrm{~Hz}$. Individual $\mathrm{SPNs}$ respond heterogeneously to change of FSI activity while the mean rate does not change significantly. $\mathbf{h}$ ) Scatter plot of change in SPN firing rate (as described in part g) as a function of number of incoming FSI connections. The change in firing rate of an SPN is strongly correlated with the number of its afferent FSI connections. 


\section{Discussion}

GABAergic synaptic currents show hyperpolarizing or depolarizing effect depending on neuronal identity, brain region, developmental stage and pathological condition. Intuitively, sufficiently low (high) GABA reversal potentials can explain the inhibitory (excitatory) effect of GABA, as GABA drives the membrane potential towards its reversal potential. However, the effect of GABA on neuronal firing rate can be more subtle than the inhibition-excitation dichotomy. In this paper, we used a leaky integrate and fire model to quantify different effects of GABA and provided a phase diagram of GABA effects. In particular, we showed that as GABA reversal potential increases, there exists a non-monotonic regime in between inhibitory and excitatory regimes, in which small GABAergic currents have an excitatory effect on firing rate, while large ones inhibit the firing rate of the postsynaptic neuron. In other words, in the non-monotonic regime, GABA can be inhibitory or excitatory depending on its strength The non-monotonic regime appears when the GABA reversal potential is below, but sufficiently close to the firing threshold. We studied the effects of GABA in the presence of input noise. We found that the non-monotonic region expands with increasing noise level, showing that fluctuations in synaptic input make the non-monotonic effect stronger and present in a wider range of GABA reversal potentials. We also showed that this non-monotonic effect is qualitatively observed in a more realistic neuronal model that captures some of the electrophysiological properties of striatal spiny projection neurons. Using simulations of a network model of local striatal circuit, we further showed that non-monotonicity can be observed in population-level dynamics. In addition, we showed that in the network model effects of changing FSI firing rates on SPNs are strongly heterogeneous.

Our findings at both single neuron and population levels can explain apparently paradoxical effects reported in the experimental literature. In the adult striatum, GABA reversal potentials are believed to be relatively high in spiny projection neurons (SPNs) 25 35, 36, which could be due to low levels of the KCC2 co-transporter expression in this cell type 37. This high GABA reversal potential can explain several results in in vitro preparations: In particular, why GABA synaptic inputs can sometimes potentiate the response of SPNs to glutamatergic inputs, depending on the relative timing between the two current types [36, and also why pharmacological inhibition of FSIs can reduce such responses 14. However, results of in vivo experiments are not consistent with a purely excitatory or inhibitory effect of GABAergic inputs from FSIs. One of the most puzzling results come from the study of [18, in which they found that both optogenetic activation and inactivation of FSIs inhibit SPN population activity. While this observed non-monotonicity might be due to experimental artifacts [38] or disynaptic effects [18, our results show that it can be arise thanks to the non-monotonic dependence of neuronal firing rate on GABA conductance. In particular, this would be consistent with the hypothesis that the striatum is set in a state close to the peak of the firing rate vs GABA conductance curve (see Fig 6e), so that both increasing and decreasing FSI activity leads to a reduction of average SPN firing rates. Note however that other studies have failed to reproduce the reduction of population SPN firing rate when FSI activity is decreased, observed in 18. In fact, multiple pharmacological, chemogenetic and optogenetic studies have found that inhibition of FSIs can disinhibit SPN population activity in vivo [14,21]. Interestingly, these studies also found that the effects of FSI inhibition on single SPNs are heterogeneous. FSI inhibition leads to both positive and negative SPN activity modulations for different sunsets of SPNs (about $60 \%$ of SPNs are disinhibited, while the other $40 \%$ are inhibited [21]. This ratio is $74 \%$ to $26 \%$ in another study [14]). This strong heterogeneity of effects can be explained in our network model by non-monotonic effects of FSI in which SPNs can be excited or inhibited depending on their FSI GABAergic inputs. Thus, our model can reproduce

311 312 313 314 315 316 317 318 319 320 321 322 323 324 325 326 327 328 329 330 331 332 333 334 335 336 337 338 339 340 341 342 343 344 345 346 347 348 349 350 351 352 353 354 355 356 357 358 359 360 361 
the diversity of effects seen in the striatal studies.

Effects of GABA conductance on neuronal firing rate that differ from pure excitation or pure inhibition have also been observed in other cell types and model studies. A non-monotonic dependence of firing rate on GABA conductance has been found in vitro in hippocampal CA1 stratum radiatum interneurons [17. These authors found that increasing GABAergic conductance first leads to an increase of firing rate, but eventually to a decrease at sufficiently high conductance, and reproduced the effect using a Hodgkin-Huxley type model. Morita et al. used a two-compartmental Hodgkin-Huxley type model, introduced by Wilson 39, with periodic GABAergic synaptic inputs and showed that a similar non-monotonic effect of GABAergic inputs on neuronal firing can be obtained 40,41. Wu et al. used a striatal circuit model in which SPNs have an elevated GABA reversal potential, and found that excitatory and inhibitory GABA currents can coexist 42 . In all these models, mixed inhibitory-excitatory effects of GABA are related to a depolarized GABA reversal potential compared to resting membrane potential. Our study complements these previous studies in several ways: First, we used a simplified model that allowed us to analytically characterize the different GABA effects and dissect the mechanisms and parameter space of the non-monotonic effect. Second, we investigated steady states with stationary inputs, rather than transient dynamics or periodic inputs as in some of the previous studies 41. Third, we systematically analyzed the role of input noise and how it influences the effects of GABA on neuronal firing. Additionally, we speculate that as many neuronal types in several brain regions undergo a reduction in GABA reversal potential through development and neurogenesis [1,8], this non-monotonic effect should have important effects at specific developmental stage and may play a role in network formation and neural integration into an existing network.

In conclusion, the results presented in this paper can explain several experimentally observed paradoxical effects of GABAergic synaptic currents and provide a framework to classify the different effects of GABA on neuronal firing rate, as a function of single neuron and network parameters. We suggest that the dichotomous framework of inhibition-excitation for GABAergic currents does not capture the full spectrum of GABA effects, as it ignores non-monotonic effects that could potentially be relevant in several brain regions. These findings have potential implications on understanding brain development, neural network formation and neural dynamics, in both normal and pathological conditions.

\section{Acknowledgments}

This work was supported by NIH BRAIN R01NS110059.

\section{References}

1. Ben-Ari Y. The GABA excitatory/inhibitory developmental sequence: a personal journey. Neuroscience. 2014;279:187-219.

2. MacKenzie G, Maguire J. Chronic stress shifts the GABA reversal potential in the hippocampus and increases seizure susceptibility. Epilepsy research. 2015;109:13-27.

3. Stein V, Nicoll RA. GABA generates excitement. Neuron. 2003;37(3):375-378.

4. Chavas J, Marty A. Coexistence of excitatory and inhibitory GABA synapses in the cerebellar interneuron network. Journal of Neuroscience.

2003;23(6):2019-2031. 
5. Enna SJ. The GABA receptors. In: The GABA receptors. Springer; 2007. p. $1-21$.

6. McCormick DA. GABA as an inhibitory neurotransmitter in human cerebral cortex. Journal of neurophysiology. 1989;62(5):1018-1027.

7. Ben-Ari Y. Excitatory actions of gaba during development: the nature of the nurture. Nature Reviews Neuroscience. 2002;3(9):728-739.

8. Ge S, Pradhan DA, Ming Gl, Song H. GABA sets the tempo for activity-dependent adult neurogenesis. Trends in neurosciences. 2007;30(1):1-8.

9. Ben-Ari Y, Khalilov I, Kahle KT, Cherubini E. The GABA excitatory/inhibitory shift in brain maturation and neurological disorders. The Neuroscientist. 2012;18(5):467-486.

10. Kirmse K, Kummer M, Kovalchuk Y, Witte OW, Garaschuk O, Holthoff K. GABA depolarizes immature neurons and inhibits network activity in the neonatal neocortex in vivo. Nature communications. 2015;6(1):1-13.

11. Van den Pol AN, Obrietan K, Chen G. Excitatory actions of GABA after neuronal trauma. Journal of Neuroscience. 1996;16(13):4283-4292.

12. Gulledge AT, Stuart GJ. Excitatory actions of GABA in the cortex. Neuron. 2003;37(2):299-309.

13. Lee J, Woo J, Favorov OV, Tommerdahl M, Lee CJ, Whitsel BL. Columnar distribution of activity dependent gabaergic depolarization in sensorimotor cortical neurons. Molecular brain. 2012;5(1):1-12.

14. O'Hare JK, Li H, Kim N, Gaidis E, Ade K, Beck J, et al. Striatal fast-spiking interneurons selectively modulate circuit output and are required for habitual behavior. Elife. 2017;6:e26231.

15. Sun YG, Wu CS, Renger JJ, Uebele VN, Lu HC, Beierlein M. GABAergic synaptic transmission triggers action potentials in thalamic reticular nucleus neurons. Journal of Neuroscience. 2012;32(23):7782-7790.

16. Haam J, Popescu IR, Morton LA, Halmos KC, Teruyama R, Ueta Y, et al. GABA is excitatory in adult vasopressinergic neuroendocrine cells. Journal of Neuroscience. 2012;32(2):572-582.

17. Song I, Savtchenko L, Semyanov A. Tonic excitation or inhibition is set by GABA A conductance in hippocampal interneurons. Nature communications. 2011;2(1):1-10.

18. Lee K, Holley SM, Shobe JL, Chong NC, Cepeda C, Levine MS, et al. Parvalbumin interneurons modulate striatal output and enhance performance during associative learning. Neuron. 2017;93(6):1451-1463.

19. Bazhenov M, Timofeev I, Steriade M, Sejnowski T. Self-sustained rhythmic activity in the thalamic reticular nucleus mediated by depolarizing GABA A receptor potentials. Nature neuroscience. 1999;2(2):168-174.

20. Jeong HY, Gutkin B. Synchrony of neuronal oscillations controlled by GABAergic reversal potentials. Neural Computation. 2007;19(3):706-729. 
21. Gittis AH, Leventhal DK, Fensterheim BA, Pettibone JR, Berke JD, Kreitzer AC. Selective inhibition of striatal fast-spiking interneurons causes dyskinesias.

Journal of Neuroscience. 2011;31(44):15727-15731.

22. Berke JD. Functional properties of striatal fast-spiking interneurons. Frontiers in systems neuroscience. 2011;5:45.

23. Fourcaud-Trocmé N, Hansel D, Van Vreeswijk C, Brunel N. How spike generation mechanisms determine the neuronal response to fluctuating inputs. Journal of neuroscience. 2003;23(37):11628-11640.

24. Hjorth JJ, Kozlov A, Carannante I, Nylén JF, Lindroos R, Johansson Y, et al. The microcircuits of striatum in silico. Proceedings of the National Academy of Sciences. 2020;117(17):9554-9565.

25. Tepper JM, Koós T, Wilson CJ. GABAergic microcircuits in the neostriatum. Trends in neurosciences. 2004;27(11):662-669.

26. Ding J, Peterson JD, Surmeier DJ. Corticostriatal and thalamostriatal synapses have distinctive properties. Journal of Neuroscience. 2008;28(25):6483-6492.

27. Taverna S, Ilijic E, Surmeier DJ. Recurrent collateral connections of striatal medium spiny neurons are disrupted in models of Parkinson's disease. Journal of Neuroscience. 2008;28(21):5504-5512.

28. Gittis AH, Nelson AB, Thwin MT, Palop JJ, Kreitzer AC. Distinct roles of GABAergic interneurons in the regulation of striatal output pathways. Journal of Neuroscience. 2010;30(6):2223-2234.

29. Siegert AJ. On the first passage time probability problem. Physical Review. 1951;81(4):617.

30. Amit DJ, Tsodyks M. Quantitative study of attractor neural network retrieving at low spike rates: I. Substrate - spikes, rates and neuronal gain. Network: Computation in neural systems. 1991;2(3):259-273.

31. Richardson MJ, Gerstner W. Synaptic shot noise and conductance fluctuations affect the membrane voltage with equal significance. Neural computation. 2005;17(4):923-947.

32. Sanzeni A, Histed MH, Brunel N. Emergence of irregular activity in networks of strongly coupled conductance-based neurons. arXiv preprint arXiv:200912023. 2020;.

33. Ermentrout GB, Terman DH. Mathematical foundations of neuroscience. vol. 35. Springer Science \& Business Media; 2010.

34. Gertler TS, Chan CS, Surmeier DJ. Dichotomous anatomical properties of adult striatal medium spiny neurons. Journal of Neuroscience.

2008;28(43):10814-10824.

35. Jiang Z, North R. Membrane properties and synaptic responses of rat striatal neurones in vitro. The Journal of Physiology. 1991;443(1):533-553.

36. Bracci E, Panzeri S. Excitatory GABAergic effects in striatal projection neurons. Journal of neurophysiology. 2006;95(2):1285-1290. 
37. Tapia D, Suárez P, Arias-García MA, Garcia-Vilchis B, Serrano-Reyes M, Bargas $\mathrm{J}$, et al. Localization of chloride co-transporters in striatal neurons. NeuroReport. 2019;30(6):457-462.

38. Owen SF, Liu MH, Kreitzer AC. Thermal constraints on in vivo optogenetic manipulations. Nature neuroscience. 2019;22(7):1061-1065.

39. Wilson HR. Simplified dynamics of human and mammalian neocortical neurons. Journal of theoretical biology. 1999;200(4):375-388.

40. Morita K, Tsumoto K, Aihara K. Possible effects of depolarizing GABAA conductance on the neuronal input-output relationship: a modeling study. Journal of neurophysiology. 2005;93(6):3504-3523.

41. Morita K, Tsumoto K, Aihara K. Bidirectional modulation of neuronal responses by depolarizing GABAergic inputs. Biophysical journal. 2006;90(6):1925-1938.

42. Wu Z, Guo A, Fu X. Generation of low-gamma oscillations in a GABAergic network model of the striatum. Neural Networks. 2017;95:72-90. 